\title{
Stem Cells - Haemobiology and Clinical Data Summarising: a Critical Review
}

\author{
Bela Balint, ${ }^{1,2}$ Mirjana Pavlovic, ${ }^{3}$ Milena Todorovic ${ }^{4,5}$
}

\begin{abstract}
Stem cells (SC) are the unique and "key-cells" in the human body "working" as a source of producing a large number (proliferation) of mature (differentiation) cells inside different tissues ("cytopoiesis") - while at the same time maintaining the ability to "reproduce" themselves (self-renewal). These events are balanced by interactive signals from the extracellular matrix, as well as microenvironment provided by stromal cells. On the other hand, SC plasticity (so-called "inter-systemic plasticity") is the ability of the most "primitive" (immature) adult SCs to switch to novel identities. The phrase SC plasticity also involves phenotypic potential of these cells, broader than spectrum of phenotypes of differentiated cells in their original tissues. Recent increasing clinical use of cell-mediated therapeutic approaches has resulted in enlarged needs for both, higher quantity of SCs and improved operating procedures during extracorporeal manipulations. The aim of harvesting procedures is to obtain the best SC yield and viability. The goal of optimised cryopreservation is to minimise cellular thermal damages during freeze/thaw process (cryoinjury). Despite the fact that different SC collection, purification and cryopreservation protocols are already in routine use - a lot of problems related to the optimal SC extracorporeal manipulations are still unresolved. The objective of this paper is to provide an integral review of early haemobiological and cryobiological research in the unlimited "SC-field" with emphasis on their entities, recent cell-concepts, extracorporeal manipulative and "graft-engineering" systems. Their therapeutic relevance and efficacy in "conventional" SC transplants or regenerative medicine will be briefly summarised. Finally, in this paper original results will not be pointed out - related to neither SC transplants nor regenerative medicine - but a light will be shed on some of them.
\end{abstract}

Key words: Stem cells; Collection; Processing; Cryopreservation; Transplant.
(1) Institute of Cardiovascular Diseases "Dedinje", Belgrade, Serbia.

(2) Serbian Academy of Sciences and Arts, Belgrade, Serbia.

(3) Department of Computer and Electrical Engineering and Computer Science, Florida Atlantic University, Boca Raton, FL, USA.

(4) Clinic for Haematology of CCS, Belgrade, Serbia

(5) Faculty of Medicine, University of Belgrade, Serbia

Correspondence:

BELA BALINT

E: bela.balint@sanu.ac.rs

\section{ARTICLE INFO}

Received: 20 December 2020 Accepted: 23 December 2020

\section{Introduction}

\section{- general considerations}

Generally, the "cytopoiesis" - defined as in vivo cell development and expansion - is a multi-cyclic process in which a variety of large quantity of mature cells are produced from a small number of stem cells (SCs). ${ }^{1-3}$ Extremely "primitive" (immature) SCs could be characterised as cells having more or less "limitless", but well-balanced self-renewal ability (SCs dividing to make more identical cells), high differentiation and proliferative capacity, as well as potential for "inter-systemic plasticity" (a possibility to "switch" into other cell lineages trough transdifferentiation). ${ }^{2-10}$

Permanent activity of SCs guarantees the "steadystate" homeostasis (from self-renewal to maturation in various "tissue-generating" (such as hae- 
matopoietic) systems. However, the concept/ event of SC aging results in a gradual degradation (up to missing) of some tissue functions - including decreased cell/tissue regenerative (renewal) potential by consequent reduction of the organ repair capacity. In clinical settings, SCs allow the repopulation of bone marrow (BM) ("conventional" SC transplants) or organ repair/restore (regenerative medicine) following therapeutic use..$^{3-6}$

Haematopoiesis is an eventful and multifarious continuous haemobiological process during which a spectrum of mature blood and immune-system cells are produced from immature compartment of haematopoietic SCs (HSCs). HSCs are actually the best evaluated and explained SCs. ${ }^{1-5}$ They are distributed in different haematopoietic compartments throughout the body during foetal and adult life. In adults, HSCs are located primarily in the BM and have full-size potential for differentiation into pluripotent or committed progenitors and finally deliver different mature blood cells required for daily blood turnover and for fighting infections. The HSC population expresses CD34 antigen - it was initially characterised as a glycoprotein expressed on haematopoietic stem/progenitor cells - thus they are named also as CD34+ cells. The CD34 is the cluster designation given to a marker present on HSC surface, but also on some "tissue-specific" stromal cells. ${ }^{1,9-13}$

Haematopoiesis is regulated through the extracellular matrix and different microenvironmental derived stromal cells by production of interactive matters and factors, such as various haematopoietic "growth factors". A number of studies have verified that a complex network of cytokines and other mediators regulate the SC survival (self-renewal), maturation (differentiation) and extensive cell production (proliferation). ${ }^{1,5-8}$

Compared to adult SCs from other tissues, HSCs are relatively not difficult to obtain, as they can be either aspirated directly from the BM or stimulated (mobilised) to move into the peripheral blood (PB) where they can be harvested by apheresis. In practice, HSCs can be collected by multiple aspirations or by harvesting from PB following application of "mobilising regimen": the use of chemotherapy and/or recombinant granulocyte-colony-stimulating-factor (rHuG-CSF) ${ }^{1-10}$ Umbilical cord blood (UCB) derived SCs can be collected using purification methods such as cell processing and rarely immunomagnetic selection. UCB transplants have provided hopeful results firstly in paediatric patients - when a matched related or unrelated BM or PB donor was unavailable..$^{1-3,14-23}$ Some marrow damages caused by the use of chemotherapy (autologous transplant setting) could be a restrictive factor of successful SC harvesting from PB ("poor mobilisers"). Namely, the quantity of total CD34+ cells in the PB mobilised healthy donors is typically higher than in the circulation of treated patients. ${ }^{10}$ That could be a critical factor for delayed haematopoietic reconstitution following autologous vs allogeneic SC transplants. ${ }^{3,10}$ However, in the bloodstream of mobilised patients the SC population is typically more "primitive" - since they have superior CD90 expression (a specific marker for immature $\mathrm{CD} 34^{+} / \mathrm{CD} 90^{+}$population or "repopulating" SCs). ${ }^{9-10}$ The presence of these cells in graft is essential for complete, stable and longterm marrow repopulation and following haematopoietic reconstitution.

The most attractive quality of extremely "primitive" adult SCs (including some HSC partitions) is the mentioned "inter-systemic plasticity" (mediated by "dedifferentiation" and "transdifferentiation" activity or "cell-reprogramming") due to the cell ability to convert or switch into different somatic cellular lineages. In appropriate conditions, they can deliver osteocytes, cardiomyocytes, hepatocytes, endothelial and other "tissue-specific" somatic cells. - $^{5-14}$ This HSC ability (similar to mesenchymal SCs) emphasizes their potential role in a spectrum of different clinical scenarios. Namely, the most "primitive" SCs open novel viewpoints and perspectives in the treatment of different diseases. The possibility of implantation of these cells into human body by reason of repair of damaged organs (regenerative medicine) and/or tissue/organ replacement (tissue engineering) will be highlighted. ${ }^{1-3,}$ 29-31 Although attractive, these SC-concepts are still the matter of controversy.

Finally, yet importantly, in this paper a number of evidence-based approaches, knowledge and facts of SC basic research and their clinical applications (based on the marrow repopulative and organ repair potential), as well as the existing limitations and future directions will be briefly reviewed.

Stem cell concept - embryonic vs adult cells Generally, the SC population can be divided into: (a) embryonic and (b) "tissue-specific" adult cells. Embryonic cells are truthfully able to differentiate into all cellular types or categories in human body. Current studies were verified that the most "primitive" adult SCs can have also similar (practically "unlimited") biological potentials. Precisely, adult 
SC multipotency and "inter-systemic plasticity", which have been newly discovered, could also lead to their hopeful use (autologous setting) of these cells in for tissue/organ regeneration or replacement. ${ }^{1-3,31}$

As expected, embryonic SCs are the most "promising" (by in fact "unlimited") cellular potentials, but also the most controversial ones. Numerous researchers view the zygote as the "ultimate-SC" since it can develop or differentiate into whichever cellular lineage in human body. The zygote has the highest degree of "inter-systemic plasticity" and it is referred to as an exact or authentic "totipotent" SC. 1, 2, 4

Following fertilisation, the zygote begins to divide and by the fifth-sixth day cells form the blastocyst. After that (the first week), cells begin to expand the coding sequence for specific functions, which makes isolating the SCs through the blastocyst-state, imperative. These cells (collected from blastocyst) can be cultured ex vivo into embryonic SCs. They have the capability to develop into all three tissue types (endoderm, ectoderm or mesoderm). After approximately 8 to 12 weeks, the "foetal SCs" are accumulated in the liver. Hypothetically, these "foetal SCs" (as well as the embryonic SCs) can be transplanted into an individual without being rejected because they have little or none of "immune-triggering-proteins" on cell surface. ${ }^{1-4}$

The compartment of adult SCs is at a more advanced phase of cell progress. Different adult SCs are able of making a number of identical "cell copies" (self-renewal), but they more frequently divide to make progenitor or precursor cells of some specific cell lineages. Earlier adult SCs were considered as cells non-capable of "transdifferentiating" into cell lineages of above mentioned three tissue types because they cannot be "rejuvenated-back" to an earlier cell stage - "dedifferentiation" followed by the process of "transdifferentiation". Adult SCs have been identified in different tissues/organs, but in very small quantities. Therefore, they can be detected in the BM, bloodstream, blood vessels, cornea, dental pulp, brain, skeletal muscle, skin, liver, pancreas and gastrointestinal tract, etc. ${ }^{2-4}$

Adult SCs could be described as cells having well-balanced self-renewal, differentiation and proliferative capacity, as well as potential for "inter-systemic plasticity" - that is ability to "switch" into other cell lineages. The self-renewal ability of "primitive" HSCs provides maintaining of the continuity of their population in BM under conditions of physiological haematopoietic balance ("steady state" haematopoiesis), but also in situation when it is partially disturbed. Less "primitive" populations of SCs and progenitors will give, however, only mature blood cells. ${ }^{1,2}$

Last but not least, SC (haemo)biology is one of the most attractive and dynamic topics in overall biology - not only from a methodical viewpoint, but also from the social, regulative and ethical points of view. However, it must be emphasised once more that there are still dilemmas and more or less "insurmountable" barriers regarding the research and (pre)clinical study of embryonic SCs. Namely, it is well known that some ethical aspect includes for many, strong argument that embryonic SC research, experiments or especially potential clinical use are very nearly equivalent for a negligent "manslaughter"., 2

\section{Stem cell collection/harvesting and ex vivo manipulations}

Historically, for transplants, BM was the primary SC-source, in which approximately 1-3\% of total nucleated cells (TNCs) express the CD34 antigen. ${ }^{1 \text {, }}$ ${ }^{10}$ The collection of mononuclear cells (MNCs; together with SCs) from BM is the same for an allogeneic donor as for an autologous patient. The $\mathrm{CD} 4^{+}$cells were determined also in $\mathrm{PB}$, but in enormously small ratio in the "steady-state" haematopoiesis: $0.01-0.05 \%$ of TNCs. ${ }^{10,13}$ The first SC harvestings from PB were accomplished in "steady-state" haematopoiesis, and numerous (6 to 9) apheresis procedures were required with following cryopreservation. Nowadays, SCs are harvested after "mobilising regimen" and the typical number of aphereses needed is not more than one to three. ${ }^{1-3,32}$ UCB has been used as an alternative SC source since the late 1980 s. $^{1-5,14}$ UCB has emerged as an alternative source of transplantable $\mathrm{CD} 4^{+}$cells for patients who lack adult HLAmatched SC donors. ${ }^{1-3,14,22,23}$

\section{Stem cell collection from bone marrow}

SCs are collected by multiple aspirations from the posterior and anterior iliac crest and (seldom) from sternum. The posterior iliac crest provides the richest site of BM-derived SCs. The procedure is performed under sterile conditions in the operation room, while the donor is generally anaesthetised. In order to provide of required MNC 


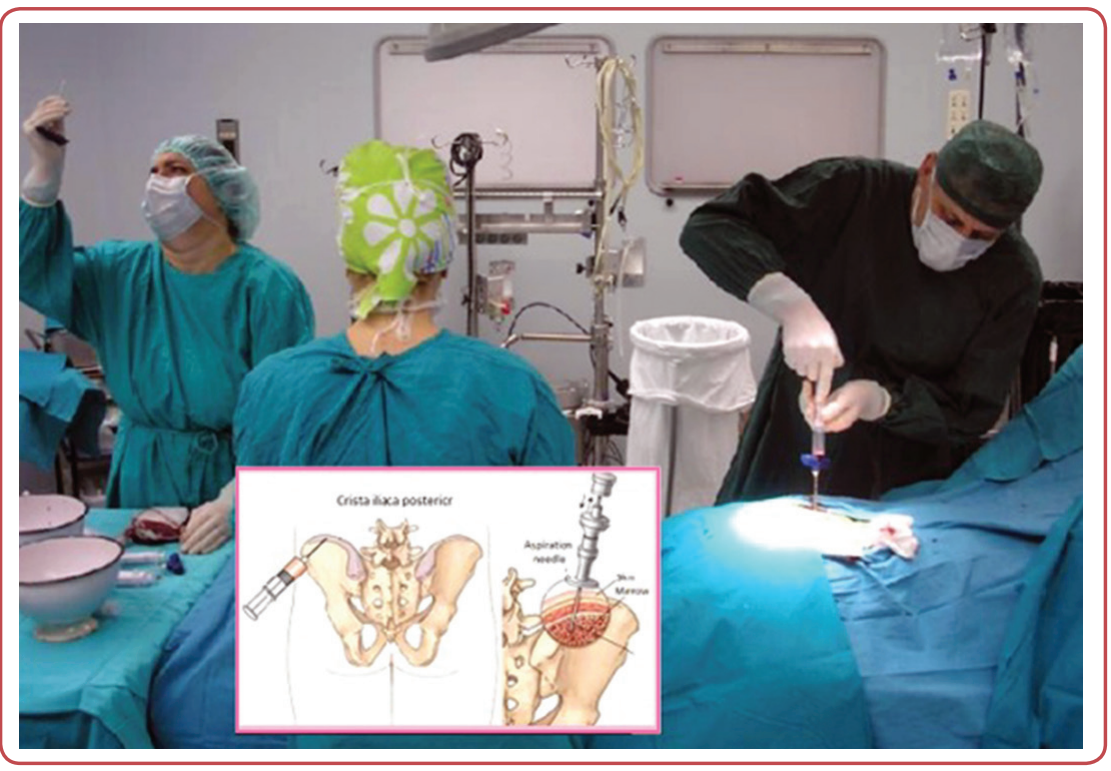

Figure 1: Stem cell collection by multiple aspirations from bone marrow

quantity ( $\geq 3 \times 10^{8} / \mathrm{kg}$ of body mass [kgbm]), approximately 200 aspirations are performed (single aspirate volume $=2-5 \mathrm{~mL}){ }^{1-3}$ Cell aspirate should be filtered in order to remove bone and lipid tissue particles and cell aggregates, as it is presented in Figure 1.

Anticoagulation is provided using citrate solution and by heparin diluted in saline (5000 IU/500 $\mathrm{mL}$ ), using autologous plasma for resuspension of collected MNCs. The highest target dose of collected marrow aspirate is $15 \mathrm{~mL} / \mathrm{kgbm}$ of donor. The volume of aspirate is relatively large (800 - 1000 $\mathrm{mL}$ ) and it contains a high count of red blood cells (RBCs) ${ }^{1-4}$ Thus, in order to prevent anaemia in donors, one unit of whole blood for autologous transfusion (to carry out during SC collection) should be collected approximately one week before cell aspirations. For ABO-incompatible (major and/ or minor) and rarely for autologous transplants (cryopreservation is required), RBC or plasma quantity reduction is obligatory (by extracorporeal processing). This method enables reduction of the aspirate volume and reduction of RBC mass ("contamination") by approximately $\geq 80 \%{ }^{4-9}$

\section{Stem cell harvesting from peripheral blood}

Nowadays, PB-derived SCs are used for approximately $80 \%$ of allogeneic and practically for all autologous SC-transplants. Transplant of SCs from PB could be characterised by a) lack of general anaesthesia and work in surgical room; b) minor harvest volume and better CD $34^{+}$yield; c) superior "engraftment-rate" and inferior transplant-related morbidity. ${ }^{1-5}$
For donor/patient anticoagulation, typically acid-citrate-dextrose formula A (ACD-A; $2.2 \%$ citrate) or rarely ACD-B (1.8\% citrate) solutions are used. The venous access is most frequently realised through ante-cubical veins (allogeneic). Autologous harvestings should be performed using central-venous catheters. These catheters have simplified cell harvesting procedure but may be associated with thrombosis of the instrumented vessels. There is also about one percent of "central-venous catheter-related" risk of local infection, pneumothorax or bleeding. ${ }^{1,33-38}$

Two harvesting procedures in the Centre for SC transplants and schematic image of "SC-hierarchy", as well as separated blood layers are presented schematically in Figure 2.

For obtaining of an acceptable SC or CD34+ yield, two standardised "mobilising regimens" are in use: a) rHuG-CSF 5 - $10 \mu \mathrm{g} / \mathrm{kgbm}$ per day (allogeneic setting) and b) rHuG-CSF $12-16 \mu \mathrm{g} / \mathrm{kgbm}$ daily combined with chemotherapy in corresponding doses (autologous setting). ${ }^{1,26,32}$

An innovative harvesting protocol to obtain sufficient SC quantity from "poor responders" or "poor mobilisers" (some patients, heavily-pretreated by radio-chemotherapy) is based on the use of AMD3100 or plerixafor (Mozobil) - which is a potent antagonist of the alpha chemokine receptor CXCR $4 .{ }^{34}$ Physiologically, the Stromal Derived Factor 1 (SDF-1), due to its interaction with CXCR4, retains $\mathrm{SCs}$ in the $\mathrm{BM}$ (phenomenon known as "homing"). Plerixafor effectively inhibits this "CXCR4-with-SDF-1" interaction. Initially, plerixafor 
Figure 2: Cell harvesting by large volume leukapheresis using the last generation of blood separators

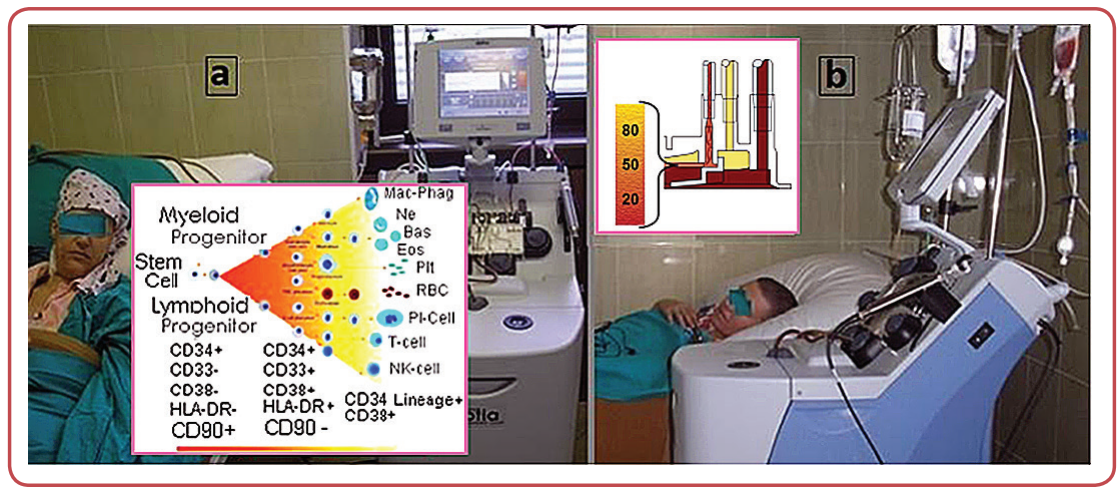

(previously known as AMD3100) was a candidate-drug for the treatment of AIDS, but it is manifested by an "unexpected side effect": white blood cell (WBC) count rise in the circulation. Thus, nowadays there are data describing superior effects of "mobilising regimen" with the use of plerixafor in combination with rHuG-CSF: increased number of progenitors and immature SCs (capable for longterm marrow repopulation - LT-HSC) in the PB and higher cell yield in the harvest. ${ }^{10,34-36}$

Determination of the optimal timing is perhaps the most critical event for both, allogeneic and autologous cell harvesting. For allogeneic donors the first apheresis is on the fifth day of rHuG-CSF application. ${ }^{1,4}$ The choice of an optimised timing for autologous harvesting is more complex and controversial - it can be determined by circulating CD34 ${ }^{+}$ cell count. Their number evidently correlates with both, collection timing and SC quantity in harvest, respectively. Exactly, it is confirmed that for a CD34 ${ }^{+} \geq 20-40 / \mu \mathrm{L}$ of patient's circulation the possibility of the $\mathrm{CD}^{2} 4^{+}$yield $\geq 2.5 \times 10^{6}$ per $\mathrm{kgbm}$ in the harvest is around 15\% after performance of one "standard" apheresis or $\geq 60 \%$ following one large volume leukapheresis (LVL). Of course, higher CD34 ${ }^{+}$number in circulation results in their superior yield in the harvest. ${ }^{1,3,4,10}$

Finally, a recent preclinical investigation has confirmed that the relative frequency of immature SC subset $\left(\mathrm{CD} 34^{+} / \mathrm{CD}^{+}{ }^{+}\right)$in $\mathrm{PB}$ possibly could be a practical and potentially more objective mobilisation predictive factor (for optimised timing) and predictor of the harvest (apheresis product) quality. ${ }^{10}$

The efficiency of harvesting can be evaluated by $\mathrm{CD} 4^{+}$quantification. In order to expect successful SC transplant, the total number of $\mathrm{CD} 34^{+}$should be about $200-300 \times 10^{6}$ cells per unit or $2-4 \times 10^{6} /$ kgbm in harvest (with platelet count $\leq 470 \times 10^{9}$ and
$\mathrm{RBC}$ volume $\leq 6-8 \mathrm{~mL}$, respectively). However, recent data support a clinical benefit associated with greater $\mathrm{CD}_{34}{ }^{+}$yield $\left(\geq 5.0 \times 10^{6} / \mathrm{kgbm}\right)$ compared to the minimum cell quantity needed $\left(\geq 1.0 \times 10^{6} /\right.$ $\mathrm{kgbm}$ ) in autologous transplant setting. The final haematocrit is $0.05-0.10$ and the final harvest volume is $200-250 \mathrm{~mL}^{1-4,32,33}$

A characteristic of $\mathrm{PB}$ harvest is a high T-lymphocyte "contamination" or rate - with consequent risk of Graft versus Host Disease (GvHD), but also a benefit from resulting Graft versus Leukaemia (GvL) effect. $^{3-6,37}$ Efficient graft purification methods, that is T-lymphocytes was developed using positive or negative immunomagnetic selection (see Figure 3).

The use of immunomagnetic system has been shown (including our own cell investigations) to be the most effective harvest purification method to achieve a $3-4 \log _{10}$ T-lymphocyte depletion while retaining around $70-80 \%$ of $\mathrm{CD}_{3} 4^{+}$cells in the graft. ${ }^{1-4}$

\section{Stem cell purification from the umbilical cord blood}

The volume of UCB unit is typically $100 \mathrm{~mL}$ (range $40-240 \mathrm{~mL}$ ) with a TNC count $\approx 1 \times 10^{9}$ and CD34 ${ }^{+}$ around $3 \times 10^{6}$ per unit. UCB can easily be cryopreserved, thus allowing for the establishment of HLA-typed SC banks. UCB is rich in "more primitive" SCs (superior BM repopulating capacity) but have the potential to transdifferentiate into non-haematopoietic cells (myocardial, neural, endothelial cells, etc). The "naive" lymphocytes in the UCB allows the use of "partially HLA-matched" grafts with no higher risk for severe GvHD. The use of UCB-derived SCs is appropriate for treatment of paediatric patients (a higher risk of graft failure was noticed in children weighing $\geq 45 \mathrm{kgbm}$ ), and for whom a matched BM or PB donor of SCs is unavailable. $^{1-4,38-41}$ 


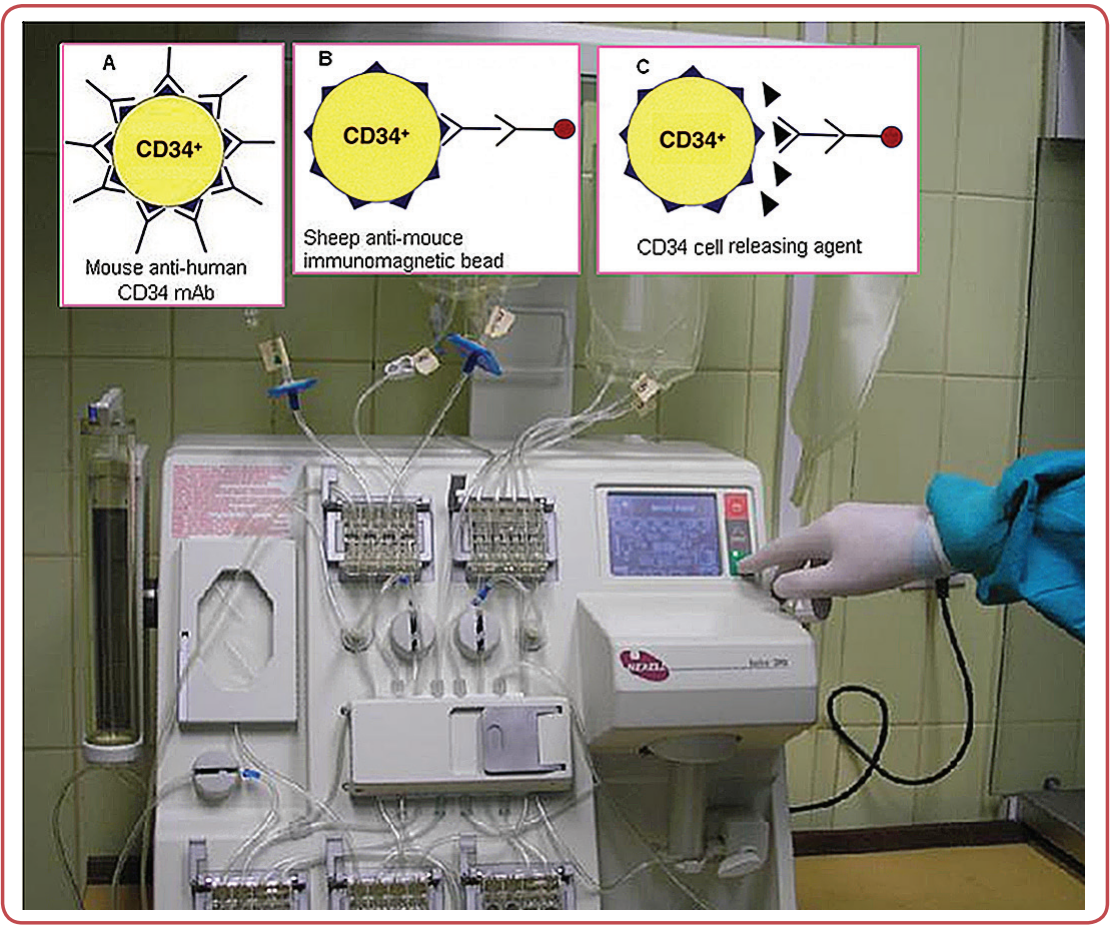

Figure 3: Immunomagnetic CD34+ selection - theory and our own application

\section{Stem cell long-term storage by cryopreser- vation}

Cryopreservation protocols (controlled-rate vs uncontrolled-rate freezing) are nowadays in routine practice - but they should be revised to minimise cell thermal damage (cryoinjury), confirming that the most effective cryopreservation system has not yet been determined. Microprocessor-restricted (controlled-rate) freezing is more effective because of superior quantitative, morphological, ultrastructural and functional cell recovery - but it requires complex equipment and high-level technical expertise. Uncontrolled-rate ("dump-freeze" without programmed cooling rate) technique is less costly because it does not require a programmed freezing device. ${ }^{42-49}$

Generally, cryoinjuries result from an extensive volume reduction (cellular dehydration or solution effect) or massive intracellular ice crystallisation (mechanical damage). Although independent, these mechanisms can also act together. The first event is expressed primarily at low-rate $\left(\leq 10^{\circ} \mathrm{C} /\right.$ min) freezing, and the second one in high-rate $\left(\geq 10^{\circ} \mathrm{C} / \mathrm{min}\right.$ ) freezing (see Figure $4 \mathrm{a}$ ). ${ }^{2,42-45}$

Consequently, determination of a specific optimised freezing rate for each cell type should be considered. It is the cooling speed high enough to prevent cell dehydration (prevention of solution effect) and adequately low to make possible efflux of water from the cell (escaping of mechanical damages).
Cell destruction has occurred when transition period from liquid to solid phase (due to fusion heat releasing) is prolonged. Its duration is directly related to the degree of cryoinjuries - therefore, the compensation of the released fusion heat is required (see Figure 4b) during the controlled-rate freezing. ${ }^{1,3,50}$

Post-thaw cell recovery and viability are adequate only when cryoprotectants - nonpenetrating (extracellular) and penetrating (intracellular) compounds - are present in the "cryobiosystem". Cryoprotective agents reduce the degree of cryoinjuries through reduction of cell dehydration and/or by decreasing the intracellular crystallisation. For SC, progenitor and mature blood cell cryopreservation, glycerol, dimethyl sulphoxide (DMSO) and hydroxyethyl-starch (HES) are regularly used, although in different concentrations..$^{1-4}$, 43, 46

In practice, SC cryopreservation consists of the following steps: (a) graft purification (if it is needed); (b) equilibration (cell exposure to cryoprotectant) and freezing; (c) cell storage at $-90 \pm 5 \stackrel{\circ}{\circ}$ (mechanical freezer), at temperature from $-120 \stackrel{\circ}{ } \mathrm{C}$ to $-150{ }^{\circ} \mathrm{C}$ (mechanical freezer or steam of nitrogen) or at $-196{ }^{\circ} \mathrm{C}$ (liquid nitrogen); and (d) cell thawing in a water bath at $37 \pm 3{ }^{\circ} \mathrm{C} .{ }^{1-6}$

The initial cryoinvestigation (using animal model) has been confirmed that the recovery of col- 
Figure 4: Cryoinjuries and our original fivestep controlled-rate freezing protocol

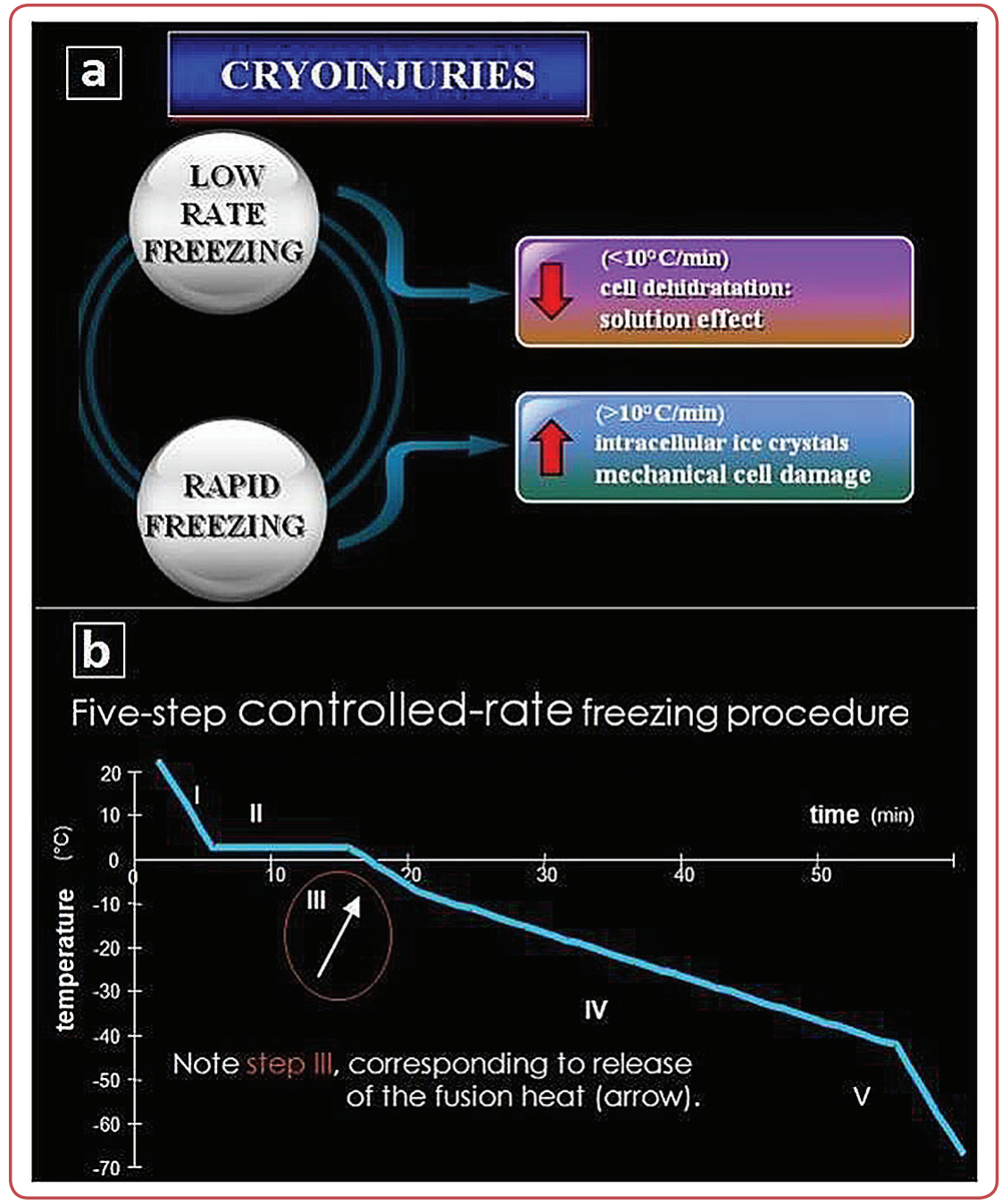

ony-forming unit spleen or pluripotent progenitors (CFU-Sd12; see Figure 5) and colony-forming unit-granulocyte macrophage or committed progenitors (CFU-GM) in the presence of $5 \%$ DMSO is superior. ${ }^{50}$

However, it has also been demonstrated that the recovery of "very-primitive" pluripotent progenitors or SCs (Marrow Repopulating Ability - MRA) is better when $10 \%$ DMSO is used. ${ }^{52}$ These results imply a different "cryobiological request" of MRA cells in comparison with the mature TNCs and progenitors.

\section{Stem cell clinical use - doctrine vs practice} The use of high-dose (radio)chemotherapy followed by allogeneic (HLA-matched related/unrelated and haploidentical), syngeneic or autologous SC transplants is considered as standard treatment for various malignant haematological and some benign, predominantly immune-mediated disorders or diseases (eg, multiple sclerosis). A similar procedure with reduced-intensity con- ditioning (RIC) can be offered to patients who are disqualified for high-dose (radio)chemotherapy because of their age or co-morbidity. ${ }^{1-6,30}$

The use of SCs for organ repair (damaged myocardium, liver, pancreas, etc) opens new perspectives in regenerative medicine. The most common haematological malignancies treated by SCs transplants are different leukaemias (nowadays mainly acute myeloid leukaemia and acute lymphoblastic leukaemia), Hodgkin's disease or non-Hodgkin's lymphoma, multiple myeloma and myelodysplastic disorders. In addition, a number of neoplasms and non-malignant diseases were treated with SC transplants such as Wilms' tumour, neuroblastoma and severe aplastic anaemia, severe combined immunodeficiency, as well as various congenital or autoimmune disorders (multiple sclerosis, immune-mediated enteropathy). ${ }^{4,5,51-55}$

Specific clinical aspects - such as optimal transplant timing, therapeutic efficacy, complications - of the treatment of these disorders will not be discussed in this article. 


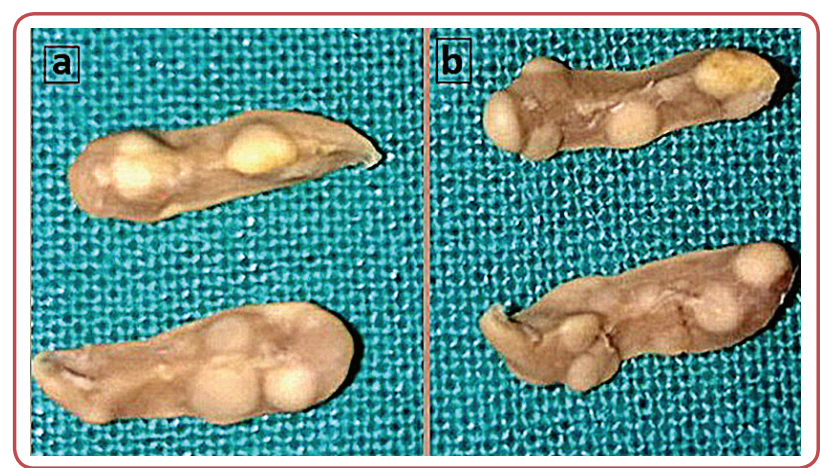

Figure 5: CFU-Sd12 cryopreserved using our controlled-rate freezing and by dimethyl sulphoxide (DMSO)

Finally, PB-derived SCs can have also a specific anti-tumour (eg, anti-leukaemia) effect, particularly in haemato-oncology. The administration of the donor-specific lymphocytes (DSLs) results frequently in an immune-mediated anti-tumour or effect. Recently systems to "separate" the GVL effect from GvHD have been developed. The best results were obtained in treatment of chronic myeloid leukaemia (CML). ${ }^{1-4,37}$ Our investigations of the use of DSLs also confirmed efficacy of this treatment in patients with Philadelphia-positive CML, relapsed after SC transplant. At the same time, our original in vitro test (named as "Test of Mixed Progenitors") was introduced to predict the clinical outcome of DSL-treatment. ${ }^{37}$

As mentioned, a number of experiments and clinical studies over the last several decades have raised the possibility that SCs from one tissue may be able to give rise to cell lineages of a completely different tissue ("inter-systemic plasticity"). This concept has been revised by the Ratajczak's group. ${ }^{31}$ They developed and confirmed the novel concept of Very Small Embryonic Like Cell (VSEL) - shown to be SCs in BM and in non-haematopoietic compartment, committed to differentiate into some other tissues. ${ }^{1-4,31}$
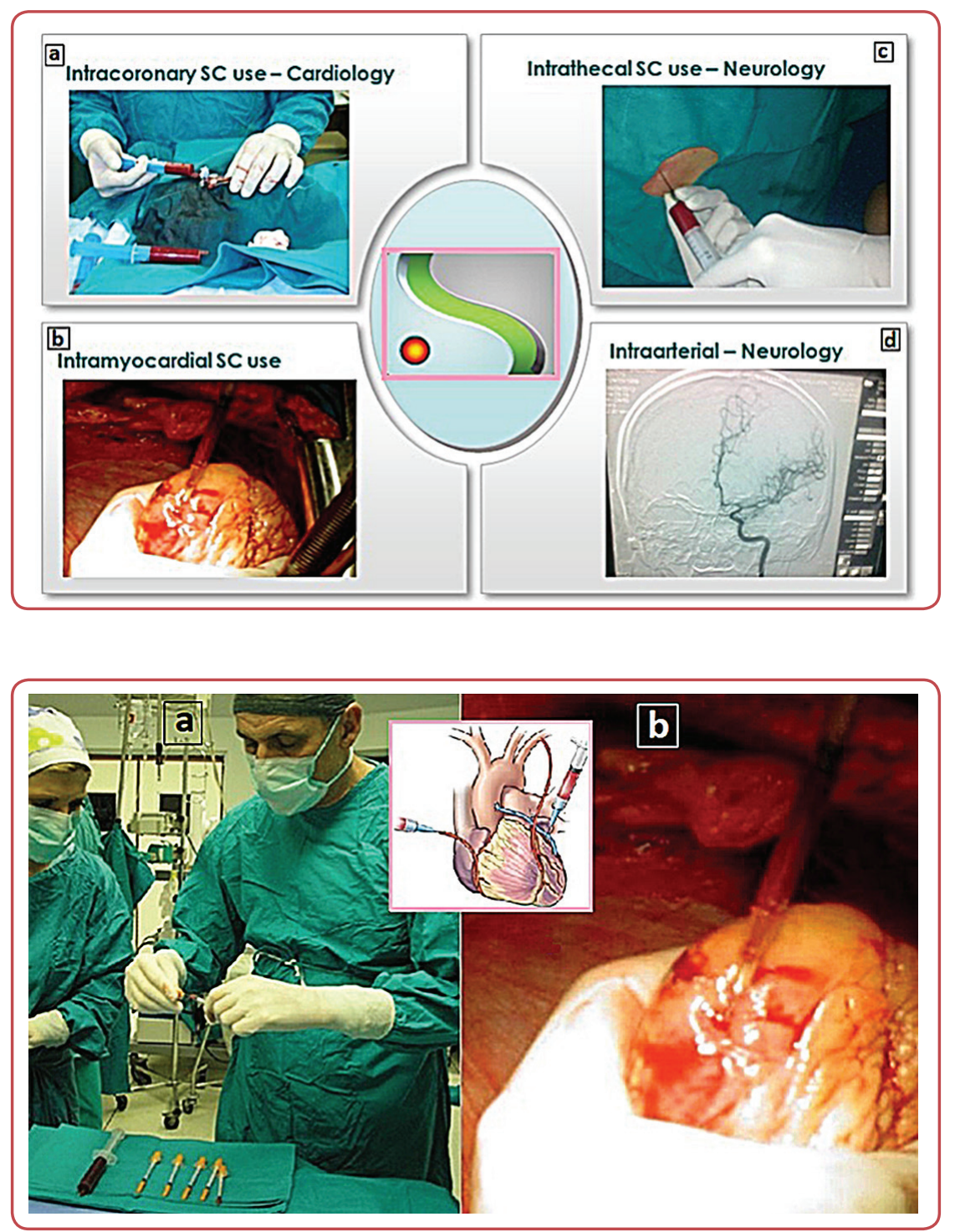

Figure 6: The application of stem cells in the fields of regenerative medicine in our center
Figure 7: Intracoronary and intramyocardial use of bone marrow-derived mononuclear cells/stemm cells 
Thanks to their "inter-systemic plasticity", therapeutic use of SCs is justified in the treatment of patients with cardiac, liver, pancreatic, brain and some other organ/tissue damages (Figure 6).

Clinical studies show that application of SCs into the damaged or ischaemic regions of myocardium results in "homing" and consequent "transdifferentiation" into cardiac cells. The presence of a significant quantity of cardiac cells is confirmed in proliferative state in peri-infarction region. Thus, the first source of these "regenerative cells" is maybe cardiac SCs, which are in the inactive stage in "intact" myocardium. Following infarction, they differentiate into mature cardiomyocytes, smooth muscle and endothelial cells. Besides, after infarction, myocardial ischaemia initiates the release of some cytokines and chemokines that induce SC recruitment from other "niches" and their "homing" into the damaged myocardium., 24-30 The in-

\section{Conclusion}

The intensification of myeloablative therapy with SC rescue and increase in the use of allogeneic transplants, as well as cell-mediated therapeutic approaches have resulted in higher needs for both, SCs and a practical ex vivo operating procedure in order to minimise cell damages. The use of "conventional" SC transplant is an imperative for treatment of haemato-oncological, some benign blood or autoimmune disorders. The concept "inter-systemic plasticity" of very "primitive" SC enables their ever-increasing therapeutic use for organ repair. However, future randomised, controlled and larger clinical trials are needed to determine the exact arrangement of this innovative treatment in the fields of regenerative medicine.

\section{References}

1. Pavlovic M, Balint B. Stem Cells and Tissue Engineering. New York: Springer; 2013.

2. Pavlovic M, Balint B. Bioengineering and cancer stem cell concept. New York: Springer; 2015.

3. Balint B, Pavlovic M, Todorovic M. From nucleated to ex vivo manipulated stem cells - an updated biological and clinical synopsis. Med Word 2020;1:1-9. tracoronary use and intramyocardial injection of MNC/SCs in the Centre is started in 2003 and in 2006, respectively (see Figure 7). ${ }^{26,29,30}$

Data related to SC use in regenerative medicine are only partially explained. There are numerous unanswered questions considering SC therapy of the ischaemic heart disease. Which mechanisms enable SCs "transdifferentiation" or "cell-reprogramming" (mediated by "extrinsic" or "intrinsic" factors)? What is the precise mechanism and level of medical benefit of MNC/SC infusion into the infarction-related artery? Do we need some exact $\mathrm{MNC} / \mathrm{SC}$ compartment and some processing or selection manner of BM before the cell inoculation into the myocardium? Are "growth factors" (cytokine) priming of MB and/or myocardium to increase the SC yield and to improve "homing" capacity of these cells into the ischaemic myocardium needed?

\section{Acknowledgements}

None.

\section{Conflict of interest}

None.

4. Balint B, Todorovic M, Pavlovic M. Stem cells - hemobiological events and clinical applications. Mac Med Rewiev 2019;73:19-22.

5. Balint B, Obradovic S, Todorovic M, Pavlovic M, Mihaljevic B. Stem cell-based (auto)grafting: from innovative research toward clinical use in regenerative medicine. In: Alimoghaddam K, editor. Stem cell biology in 
normal life and diseases. London: InTechOpen; 2013. p. 111-35.

6. Barriga F, Ramírez P, Wietstruck A, Rojas N. Hematopoietic stem cell transplantation: clinical use and perspectives Biol Res 2012;45:307-16.

7. Singh AK, McGuirk JP. Allogeneic stem cell transplantation: A historical and scientific overview. Cancer Res 2016;76:6445-51.

8. Balassa K, Danby R, Rocha V. Haematopoietic stem cell transplants: principles and indications. Br J Hosp Med 2019;80:33-39.

9. Balint B, Stamatovic D, Todorovic M, Elez M, Vojvodic D, Pavlovic M, Cucuz-Jokic M. Autologous transplant in aplastic anemia: quantity of CD34+/CD90+ subset as the predictor of clinical outcome. Transfus Apher Sci 2011;45:137-41.

10. Balint B, Stanojevic I, Todorovic M, Stamatovic D, Pavlovic M, Vojvodic D. Relative frequency of immature CD34+/CD90+ subset in peripheral blood following mobilization correlates narrowly and inversely with the absolute count of harvested stem cells in multiple myeloma patients. Vojnosanit Pregl 2017;74:1071-7.

11. Sumikuma T, Shimazaki C, Inaba T, Ochiai N, Okano A, Hatsuse M, et al. CD34+/CD90+ cells infused best predict late haematopoietic reconstitution following autologous peripheral blood stem cell transplantation. $\mathrm{Br}$ J Haematol 2002;117:238-44.

12. Villaron EM, Almeida J, Lopez-Holgado N, Sanchez-GuijoFM, Alberca M, Blanco B, et al. In leukapheresis products from non-Hodgkin's lymphoma patients, the immature hematopoietic progenitors show higher CD90 and CD34 antigenic expression. Transfus Apher Sci 2007;37:145-56.

13. Barnett D, Janossy G, Lubenko A, Matutes E, Newland A, Reilly JT. Guideline for the flow cytometric enumeration of CD34+haematopoietic stem cells. Clin Lab Haem 1999;21:301-8.

14. Skoric D, Balint B, Petakov M, Sindjic M, Rodic P. Collection strategies and cryopreservation of umbilical cord blood. Transfus Med 2007;17:107-13.

15. Lasky LC, Ash RC, Kersey JH. Collection of pluripotential hematopoietic cells by cytapheresis. Blood 1982;59:822-7.

16. Sato N, Sawada K, Takahashi TA, Mogi Y, Asano S, Koike $\mathrm{T}$, et al. A time course study for optimal harvest of peripheral blood progenitor cells by granulocyte colony-stimulating factor in healthy volunteers. Exp Hematol 1994;22:973-8.

17. Jamal A, Khan MT, Parveen S, Rizvi Q, Farzana T, Zaidi $\mathrm{U}$, et al. Peripheral blood stem cell harvest hpc count is an effective surrogate marker for CD34+ cell count in allogeneic stem cell transplant setting. Transl Oncol 2020;13:100788. doi:10.1016/j.tranon.2020.100788.

18. Moog R. Apheresis techniques for collection of peripheral blood progenitor cells. Transfus Apheresis Sci 2004;31:207-20.

19. Martin I, Albert A, Alcorta I, Estella J, Rives S, Toll T, et al. Large volume leukapheresis for peripheral blood stem cell collection in children under $10 \mathrm{~kg}$ in weight. Bone Marrow Transplant 2003;31:517-8.

20. Moreiras-Plaza M, Albo C, Ares C. Efficacy and safety of femoral vascular access for peripheral blood stem cell (PBSC) collection. Bone Marrow Transplant 2004;33:347-50.

21. Saif MW, Leitman SF, Cusack G, Horne M, Freifeld A, Venzon D, et al. Thromboembolism following removal of femoral venous apheresis catheters in patients with breast cancer. Ann Oncol 2004;15:1366-72.

22. Rogers I, Casper RF. Umbilical cord blood stem cells. Best Pract Res Clin Obstet Gynaecol 2004;18:893-908.

23. Cohen Y, Nagler A. Umbilical cord blood transplantation - how, when and for whom? Blood Rev 2004;18:167-79.

24. Beltrami AP, Urbanek K, Kajstura J, Yan S-M, Finato N, Bussani R et al. Evidence that human cardiac myocites divide after myocardial infarction. N Engl J Med 2001;344:1750-7.

25. Müller P, Pfeiffer P, Koglin J et al. Cardiomyocites of noncardiac origin in myocardial biopsies of human transplanted hearts. Circulation 2002;106:31-5.

26. Balint B, Stamatovic D, Todorovic M, Jevtic M, Ostojic G, Pavlovic M, et al. Stem cells in the arrangement of bone marrow repopulation and regenerative medicine. Vojnosanit Pregl 2007;64:481-4.

27. Shintani S, Murohara T, Ikeda H, Uenoi T, Honma T, Katoh A, et al. Mobilization of endothelial progenitor cells in patients with acute myocardial infarction. Circulation 2001;103:2776-9.

28. Hassink RJ, de la Rivere AB, Mummery CL, Doevendans PA. Transplantation of cells for cardiac repair. J Am Coll Cardiol 2003;41:771-7.

29. Obradovic S, Balint B, Romanovic R, Trifunovic Z, Rusovic $S$, Baskot $B$, et al. Influence of intracoronary injections of bone-marrow-derived mononuclear cells on large myocardial infarction outcome: quantum of initial necrosis is the key. Vojnosanit Pregl 2009;66:9981004.

30. Trifunovic Z, Obradovic Z, Balint B, Ilic R, Vukic Z, Sisic $M$, et al. Ischemic cardiomyopathy treated with coronary bypass surgery and concomitant intramyocardial bone marrow mononuclear cell implantation - long term follow-up study. Vojnosanit Pregl 2015;72:22532.

31. Ratajczak J, Miekus K, Kucia M, Zhang J, Reca R, Dvorak P, and Ratajczak MZ. Embryonic stem cell-derived microvesicles reprogram hematopoietic progenitors: evidence for horizontal transfer of mRNA and protein delivery. Leukemia 2006;20:847-56.

32. Balint B, Ljubenov M, Stamatovic D, Todorovic M, Pavlovic M, Ostojic G, et al. Stem cell harvesting protocol research in autologous transplantation settings: large volume vs. conventional cytapheresis. Vojnosanit Pregl 2008;65:545-51.

33. Philip J, Bajaj AK, Sharma S, Kushwaha N, Kumar S, Kumar Biswas A. Allogeneic peripheral blood stem cell transplant: correlation of donor factors with yield, engraftment, chimerism, and outcome: retrospective review of a single institute during a 3-year period. Lab Med 2020;51:362-9.

34. De Clercq E. The AMD3100 story: the path to the discovery of a stem cell mobilizer (Mozobil). BiochemPharmacol 2009;77:1655-64.

35. Fergadis E, Assi A, Kranidioti E, Kosma A, Karakosta $\mathrm{M}$, Miltiadous C, et al. Plerixafor-aided mobilization of peripheral blood hematopoietic stem cells to support subsequent high-dose chemotherapy after a prior autologous transplant. Clin Lymphoma Myeloma Leuk 2020;20:e50-e57. doi: 10.1016/j.clml.2019.11.022.

36. Lidonnici MR, Aprile A, Frittoli MC, Mandelli G, Paleari Y, Spinelli A, et al. Plerixafor and G-CSF combination mobilizes hematopoietic stem and progenitors cells with a distinct transcriptional profile and a reduced in vivo homing capacity compared to plerixafor alone. 
Haematologica 2017;102:e120-4. doi: 10.3324/haematol.2016.154740.

37. Petakov M, Balint B, Bugarski D, Jovcic G, Stojanovic N, Vojvodic D, et al. Donor leukocyte infusion - the effect of mutual reactivity of donor's and recipietnt's peripheral blood mononuclear cell on hematopoietic progenitor cells growth. Vojnosanit Pregl 2000;57:89-93.

38. Hussein E, DeFor T, Wagner JE, Sumstad D, Brunstein CG, McKenna DH. Evaluation of post-thaw CFU-GM: clinical utility and role in quality assessment of umbilical cord blood in patients receiving single unit transplant. Transfusion 2020;60:144-154

39. Chakraborty S, Gupta D, Thakral D, Bakhshi S, Kumar P, Kabra SK, et al. Successful reconstitution of leukocyte adhesion defect after umbilical cord blood stem cell transplant. Cent Eur J Immunol 2020;45:117-21.

40. Solomon SR, Martin AS, Zhang MJ, Ballen K, Bashey A, Battiwalla $\mathrm{M}$, et al. Optimal donor for africanamericans with hematologic malignancy: HLA-haploidentical relative or umbilical cord blood transplant. Biol Blood Marrow Transplant 2020;26:1930-6.

41. Mousavi SH, Zarrabi M, Abroun S, Ahmadipanah M, Abbaspanah B. Umbilical cord blood quality and quantity: Collection up to transplantation. Asian J TransfusSci 2019;13:79-89.

42. Polge C, Smith AU, Parkes AS. Revival of spermatozoa after vitrification and dehydratation at low temperatures. Nature 1949;164:666.

43. Lovelock JE, Bishop MWH. Prevention of freezing damage to living cells by dimethyl-sulfoxide. Nature 1959;183:1394-5.

44. Meryman HT. Mechanics of freezing in living cells and tissues. Science 1956;124:515-21.

45. Mazur P. Theoretical and experimental effects of cooling and warming velocity on the survival of frozen and thawed cells. Cryobiology 1966;2:181-92.

46. Stiff PJ, Koester AR, Weidner MK, Dvorak K, Fisher RI. Autologous bone marrow transplantation using unfractionated cells cryopreserved in dimethylsulfoxide and hydroxyethyl starch without controlled-rate freezing. Blood 1987;70:974-8.
47. Clark J, Pati A, McCarthy D. Successful cryopreservation of human bone marrow does not require a controlled-rate freezer. Bone Marrow Transplant 1991;7:121-5

48. Halle P, Tournilhac O, Knopinska-Posluszny W, Kanold J, Gembara P, Boiret N, et al. Uncontrolled-rate freezing and storage at -80 degrees $\mathrm{C}$, with only 3.5 -percent DMSO in cryoprotective solution for 109 autologous peripheral blood progenitor cell transplantations. Transfusion 2001;41:667-73.

49. McCullough J, Haley R, Clay M, Hubel A, Lindgren B, Moroff G. Long-term storage of peripheral blood stem cells frozen and stored with a conventional liquid nitrogen technique compared with cells frozen and stored in a mechanical freezer. Transfusion 2010;50:808-19.

50. Balint B, Ivanovic Z, Petakov M, Taseski J, Jovcic G, Stojanovic $\mathrm{N}$, et al. The cryopreservation protocol optimal for progenitor recovery is not optimal for preservation of MRA. Bone Marrow Transplant 1999;23:613-9.

51. Zahid U, Akbar F, Amaraneni A, Husnain M, Chan O, Riaz IB, et al. A review of autologous stem cell transplantation in lymphoma. CurrHematolMalig Rep 2017;12:217-26.

52. Takami A. Hematopoietic stem cell transplantation for acute myeloid leukemia. Int J Hematol 2018;107:513-8.

53. Todorovic Balint M, Jelicic J, Bila J, Balint B, Antic D, Trajkovic G , et al. Influence of applied CD34+ cell dose on the survival of Hodgkin's lymphoma and multiple myeloma patients following autologous stem cell transplants. Vojnosanit Pregl 2020;77(8):844-51.

54. Ahmed Z, Imdad A, Connelly JA, Acra S. Autoimmune enteropathy: An updated review with special focus on stem cell transplant therapy. Dig Dis Sci 2019;64:64354.

55. Das J, Sharrack B, Snowden JA. Autologous haematopoietic stem cell transplantation in multiple sclerosis: A review of current literature and future directions for transplant haematologists and oncologists. Curr Hematol Malig Rep 2019;14:127-35. 